\title{
Online postconcussion return-to-play instructions
}

\author{
Jennylee S. Swallow, BS, Jacob R. Joseph, MD, ${ }^{2}$ Kylene Willsey, MD, ${ }^{3}$ Andrea A. Almeida, MD, ${ }^{4}$ \\ Matthew T. Lorincz, MD, PhD, ${ }^{4}$ Paul Park, MD, ${ }^{2}$ Nicholas J. Szerlip, MD, ${ }^{2}$ and \\ Steven P. Broglio, $\mathrm{PhD}^{1}$
}

\begin{abstract}
${ }^{1}$ NeuroTrauma Research Laboratory, School of Kinesiology; Departments of ${ }^{2}$ Neurosurgery and ${ }^{3}$ Pediatrics; and ${ }^{4}$ NeuroSport, Department of Neurology, University of Michigan, Ann Arbor, Michigan
\end{abstract}

OBJECTIVE The authors of recent concussion guidelines have sought to form a consensus on injury management, but it is unclear if they have been effective in conveying this information to the public. Many parents and athletes obtain medical recommendations via the Internet. This review is aimed at evaluating consistency between online resources and published guideline statements in postconcussion return-to-play (RTP) decisions.

METHODS Five websites were selected through a Google search for RTP after concussion, including a federal government institution (Centers for Disease Control and Prevention) website, a national high school association (National Federation of State High School Associations) website, a popular nationally recognized medical website for patients (WebMD), a popular parent-driven website for parents of children who participate in sports (MomsTeam), and the website of a private concussion clinic (Sports Concussion Institute), along with a university hospital website (University of Michigan Medicine). Eight specific items from the Zurich Sport Concussion Consensus Statement 2012 were used as the gold standard for RTP recommendations. Three independent reviewers graded each website for each of the 8 recommendations $(A=$ states guideline recommendations appropriately; $B=$ mentions guideline recommendation; $C=$ does not mention guideline recommendation; $F=$ makes inappropriate recommendation).

RESULTS A grade of $A$ was assigned for $45.8 \%$ of the recommendations, $B$ for $25.0 \%$, $C$ for $25.0 \%$, and $F$ for $4.2 \%$. All the websites were assigned an A grade for the recommendation of no RTP on the day of injury. Only 1 website (WebMD) mentioned medication usage in conjunction with the Zurich statement, and only 2 websites (Sports Concussion Institute and University of Michigan Medicine) mentioned appropriate management of persistent symptoms. None of these websites commented correctly on all 8 guideline recommendations.

CONCLUSIONS Online resources are inconsistent in relaying guideline recommendations for RTP and provide a potential source of confusion in the management of concussion for athletes and their parents, which can result in inappropriate RTP decisions.

https://thejns.org/doi/abs/10.3171/2017.7.PEDS17180

KEY WORDS concussion; mild traumatic brain injury; guidelines; online resources; return to play; trauma

$\mathrm{S}$ PORTS-RELATED concussions (SRCs) account for an estimated 250,000 pediatric patients presenting to emergency departments across the United States; the overall estimated incidence is 1.6-3.8 million SRCs annually. ${ }^{6,82}$ The effective diagnosis and management of concussions have become essential for ensuring safe return to play (RTP) for athletes so as to minimize risk of longterm consequences. However, as a result of burgeoning evidence, management strategies for RTP of athletes with an SRC are continually changing. The American Medical So- ciety for Sports Medicine, the American Academy of Neurology, the National Athletic Trainers' Association, and the Concussion in Sport Group (CISG) have each released guidelines with respect to the diagnosis, management, and RTP protocol for patients with concussion..$^{5,16,18,22}$ Although these guidelines differ from each other slightly, all of them are similar and have common statements that align with current literature in the field. ${ }^{5,16,18,22,31}$ The CISG consensus statements, which provide expert opinion on concussion diagnosis and management from diverse authors, are used

ABBREVIATIONS CDC = Centers for Disease Control and Prevention; MT = MomsTeam; NFHS = National Federation of State High School Associations; RTP = return to play; $\mathrm{SCI}=$ Sports Concussion Institute; SRC = sports-related concussion; UM = University of Michigan Medicine.

SUBMITTED April 10, 2017. ACCEPTED July 21, 2017.

INCLUDE WHEN CITING Published online November 10, 2017; DOI: 10.3171/2017.7.PEDS17180. 
widely among physicians and other health care providers. The 2012 Zurich Sport Concussion Consensus Statement guidelines were published and made freely available without copyright restriction to maximize their impact among practitioners. The consensus process was described in that statement and included a systematic literature review of all available evidence. ${ }^{22}$

A central tenet of current management in each guideline is the recommendation to avoid same-day RTP because of the chance of additional head impacts, delayed symptom resolution, and, rarely, second-impact syndrome. . $^{3,11,23}$ More than $80 \%$ of athletes with SRC returned to play within 1 month of symptom onset. ${ }^{20}$ The current approach to concussion management during this time includes attention to physical and cognitive rest until acute symptom resolution and neurocognitive recovery, followed by graded return to physical activity. ${ }^{31}$ However, recent literature has suggested that a significant number of athletes might be returning to play inappropriately early. ${ }^{20}$

Although these recent guidelines were aimed at developing consensus around concussion diagnosis, acute and postacute management, and RTP protocol, it is unclear how effective they have been in influencing public opinion on concussion care. Despite the availability of health care professionals and athletic trainers as resources for athletes and their parents, potential barriers to accessing them exist. $^{14}$ The ease of access to online resources through conventional search engines is a powerful alternative influence on athlete and parent opinions. ${ }^{2}$ In this study, we sought to identify how well online resources convey the recommendations from the Fourth International Conference on Concussion in Sport held in Zurich. We hypothesized that we would find significant discrepancies between expert-opinion recommendations for RTP management and recommendations found in online resources.

\section{Methods}

A Google search was conducted in January 2017 using variations of terms for concussion, including "concussion," "mild traumatic brain injury," and "head injury." These terms were combined with search terms for RTP including "return to play" and "length of time to sit out." In addition, searches were conducted using questions such as "how long before I can return to play after a concussion?" Through this method, 5 websites were selected. These websites were selected based on their availability within the top 10 results on any specific search and those targeted to a diverse audience. These sites included a federal government institution website, a national high school association website, a popular nationally recognized medical website for patients, a popular parent-driven website for parents of children who participate in sports, and the website of a private concussion clinic., ${ }^{719,24,27,28}$ In addition, the website of our institution (an academic medical center) was included.

The 2012 Zurich guidelines were evaluated for all their recommendations related to RTP. ${ }^{22}$ We identified 8 specific recommendations (summarized in Table 1). Three members of the research team individually graded each website for the 8 criteria identified. A grade of A was assigned for
TABLE 1. RTP recommendations*

RTP should not occur on the day of injury

Neuropsychological testing can be used to assist RTP decisions

Use a graded program of exertion before medical clearance and RTP

The initial period of rest should be 24-48 hours

Each step of the graded RTP protocol should take 24 hours

For any recurrence of symptoms during the graded protocol, the patient should rest and then go back to the previous asymptomatic level

Symptoms that persist for $>10$ days should be managed in a multidisciplinary fashion

RTP should not occur while the patient is taking medication(s) for concussion symptoms

* Based on information from McCrory et al: Clin J Sport Med 23:89-117, 2013.

stating guideline recommendations appropriately, a grade of $\mathrm{B}$ was assigned if the guideline recommendation was mentioned but not complete in its description, a grade of $\mathrm{C}$ was assigned if the guideline recommendations were not mentioned, and a grade of $\mathrm{F}$ was assigned if a recommendation that did not concur with the guideline recommendation was made. For any discrepancies in grading between the raters, the majority grade was used. If 3 separate grades were assigned, the item was reviewed again, and a consensus grade was assigned. Consensus was achieved by reviewing the website as a group and determining if the criteria for a grade of $\mathrm{A}$ or $\mathrm{F}$ were met. If not, a grade of $\mathrm{B}$ was assigned if mention of the recommendation was made, and a grade of $\mathrm{C}$ was assigned if no such mention was made. Interrater variability of grading was determined by calculating the 2-way random average-measures intraclass correlation coefficient and its $95 \%$ confidence interval. Statistical analysis was performed using IBM SPSS Statistics 24 (IBM Corp.). A secondary grading scale was used to determine overall grades for each website. A grade of A was assigned 3 points, a grade of B was assigned 2 points, a grade of $C$ was assigned 1 point, and a grade of $\mathrm{F}$ was assigned 0 points. The maximum number of points available for each website was 24 .

\section{Results}

The results are summarized in Table 2. Selected for this study were the websites of the Centers for Disease Control and Prevention (CDC), the National Federation of State High School Associations (NFHS), WebMD, University of Michigan Medicine (UM), MomsTeam (MT), and the Sports Concussion Institute (SCI). Of the 6 websites, 5 (CDC, NFHS, WebMD, UM, and MT) published the date of their last revision, and all of them were revised after publication of the 2012 Zurich guidelines. The SCI did not publish its date of last revision but did provide a link to the Zurich guidelines. The interrater intraclass correlation coefficient was 0.82 (95\% CI 0.70-0.89, p < 0.001), which indicates good reliability across all recommendations. For reviewer discrepancies, a majority grade was used 12 times, and a consensus grade was used 8 times. A grade of A was assigned 22 times (45.8\%), B was assigned 12 times (25\%), $\mathrm{C}$ was assigned 12 times (25\%), and $\mathrm{F}$ was assigned 
TABLE 2. Website grades for RTP after concussion in accordance with guideline recommendations*

\begin{tabular}{|c|c|c|c|c|c|c|}
\hline \multirow[b]{2}{*}{ Guideline } & \multicolumn{6}{|c|}{ Grade Ascribed to the Website $†$} \\
\hline & MT & WebMD & NFHS & $\mathrm{SCl}$ & $\mathrm{CDC}$ & UM \\
\hline RTP should not occur on the day of injury & A & A & A & A & A & A \\
\hline Neuropsychological testing can be used to assist RTP decisions & $\mathrm{B}$ & C & C & $\mathrm{F}$ & C & A \\
\hline Use a graded program of exertion before medical clearance and RTP & A & A & $\mathrm{B}$ & A & A & A \\
\hline The initial period of rest should be $24-48$ hours & $\mathrm{F}$ & $\mathrm{B}$ & $\mathrm{B}$ & $\mathrm{B}$ & B & $\mathrm{B}$ \\
\hline Each step of the graded RTP protocol should take 24 hours & A & A & B & A & B & A \\
\hline $\begin{array}{l}\text { For any recurrence of symptoms during the graded protocol, the patient should rest and } \\
\text { then go back to the previous asymptomatic level }\end{array}$ & A & A & B & A & A & A \\
\hline Symptoms that persist for $>10$ days should be managed in a multidisciplinary fashion & C & C & C & B & A & C \\
\hline RTP should not occur while the patient is taking medication(s) for concussion symptoms & C & $B$ & $C$ & C & C & $C$ \\
\hline
\end{tabular}

* Based on information from McCrory et al: Clin J Sport Med 23:89-117, 2013.

† Grading scale: $\mathrm{A}$ = states guideline recommendations appropriately; $\mathrm{B}=$ mentions guideline recommendation; $\mathrm{C}=$ does not mention guideline recommendation; $\mathrm{F}=$ makes inappropriate recommendation.

2 times (4.2\%). Each website was given a grade of A for recommending no RTP on the day of injury. Similarly good consensus was reached for the websites recommending the use of a graded program of exertion (all graded A except for the NFHS, which was graded B), requiring 24 hours per step of the graded program (all graded A except for the NFHS, which was graded B), and for recommending management of recurrent symptoms in the graded protocol (all graded A except for the NFHS, which was graded B).

The highest overall grades were assigned to the UM (19 of 24), WebMD (18 of 24), CDC (18 of 24), and SCI (17 of $24)$ websites. The lowest overall grades were assigned to the MT (16 of 24) and NFHS (14 of 24) websites. Only 1 website (WebMD) mentioned medication usage in its RTP recommendations, only 2 websites (SCI and CDC) commented on the management of persistent symptoms, and 3 (UM, MT, and SCI) websites commented on neuropsychological testing.

\section{Discussion}

Appropriate RTP decisions are a cornerstone of concussion management, and careful consideration of readiness for RTP should be used to reduce the risk of recurrent injury. Multiple factors, including age, symptom resolution, clinical examination return to baseline, and response to graded physical activity, should be considered before allowing RTP of an athlete. ${ }^{16}$ No athlete should resume play on the day of injury, because adequate cognitive and physical rest before RTP is vital for preventing reinjury and/or prolonged symptoms. The RTP protocol recommended in the Zurich statement is a 5-step program that gradually reintroduces injured athletes to play while ensuring their safety. For athletes to begin the RTP protocol, they must be asymptomatic and not taking medication for the concussion, and they must be cleared by a licensed medical professional. ${ }^{16,18}$ Physical activity should progress in a stepwise fashion every 24 hours, provided the athlete remains symptom free. If RTP occurs prematurely, the athlete might experience decreased cognitive ability and reaction time, which could increase his or her risk of secondary injury and can result in prolonged symptoms. In severe cases, athletes can acquire second-impact syndrome or diffuse axonal swelling, which makes the gradual RTP protocol all the more important. ${ }^{18}$

Although current guidelines clearly map the RTP protocol, data from concussion surveillance systems suggest that these recommendations are not being followed; ${ }^{20}$ $3.1 \%$ of athletes returned to play within 24 hours of injury, and $20.4 \%$ of athletes returned to play within 6 days of injury, which suggests insufficient rest before they begin the RTP protocol. In 1 study, $10.1 \%$ of young athletes returned to play within 24 hours of injury, ${ }^{20}$ which is concerning given that young athletes tend to require more time for concussion recovery (on average, 17 days of rest before RTP) than do adults. ${ }^{25,30}$ These rates emphasize the need for increased awareness of the proper RTP protocol.

The reasons for accelerated RTP are unclear. One potential reason is an inability to access appropriate health care professionals. Individuals across the United States are faced with numerous barriers to accessing proper health care, including lack of time, resources, and funds. Results of previous studies have suggested that patients with public insurance return to activity sooner after concussion than those with private insurance..$^{32}$ Although all US states have passed legislation to address youth SRC, many of them do not require clearance from a medical professional. ${ }^{12,13}$ Barriers such as these have led to the Internet becoming a powerful resource from which one can access health care knowledge., ${ }^{2,10,15}$ The large number of online health resources leads to difficulty in evaluating the reliability, quality, validity, and thoroughness of a website and the information it presents. ${ }^{26}$ The spread of misinformation online is a well-known phenomenon. ${ }^{9}$ Previous studies found that the quality of online concussion-management resources varies, and the inconsistent language used can lead to confusion among the general public., ${ }^{1,4}$

Our goal for this study was to identify a variety of popular websites that might act as a resource for parents and athletes who have suffered a concussion and need to engage in an RTP protocol. These websites were then graded on the basis of the Zurich consensus statement, ${ }^{22}$ which was developed by an international multispecialty commu- 
nity of concussion experts. Recommendations against athletes returning to play on the day of injury were consistent across all resources and with those in the Zurich consensus statement. Similarly, recommendations for the use of a graded program of exertion and management of recurrent symptoms during this program were consistent across the websites. This consensus is a positive progression that highlights growing public knowledge about appropriate concussion management. Among the websites examined, however, we found numerous contradictions, confusion, and differences of opinion. For example, the MT website stated that a period of 7 days of rest and recovery is needed before beginning an RTP protocol, although this statement is not supported by the Zurich guidelines. ${ }^{28}$ One website (SCI) stated that computerized neuropsychological tests are a cornerstone of management, although the Zurich guidelines note that these tests are not substitutes for formal neuropsychological testing. ${ }^{27}$ None of the websites correctly noted all the recommendations made in the Zurich consensus statement. The use of medication to treat concussion symptoms and manage persistent concussion symptoms was a particular area of inconsistency. In fact, only 1 website (WebMD) commented on the use of medications, and only 2 websites (SCI and CDC) commented on the management of prolonged symptoms.

The results of this study suggest that online resources can be a source of confusion among parents and athletes regarding RTP strategies and might contribute to inappropriate RTP. Given these findings, we would advocate for improved communication and outreach efforts from guideline committees to help shape the practice of academic and community physicians, athletic trainers, school administrators, coaches, parents, and athletes. Although the guidelines themselves do not need to be written word for word on all websites, the basic core of the guidelines should be conveyed online in a consistent manner.

There were numerous limitations to this study. Although we obtained a representative sample of different websites, a vast number of other existing websites and online resources related to concussion management were not examined. In addition, the most recent Zurich consensus statement was published in 2013, but it will be updated in 2017. Because concussion research is a rapidly growing field, some guideline recommendations might be antiquated. For example, results of recent studies have suggested that a period of "relative rest" is likely more appropriate than complete cognitive and physical rest immediately after concussion. ${ }^{17,29}$ In this regard, online resources might be ahead of the curve rather than behind it.

\section{Conclusions}

Online resources do not provide a consistent message about concussion management. Such resources lack clarity and do not follow published guideline recommendations, which might be a result of incomplete research and education. We suggest that parents and athletes communicate their symptoms to an athletic trainer or physician to implement the RTP protocol after a concussion. A clear and concise message regarding concussion management is needed to improve overall care and proper RTP after concussion.

\section{References}

1. Ahmed OH, Sullivan SJ, Schneiders AG, McCrory PR: Concussion information online: evaluation of information quality, content and readability of concussion-related websites.

Br J Sports Med 46:675-683, 2012

2. Amante DJ, Hogan TP, Pagoto SL, English TM, Lapane KL: Access to care and use of the Internet to search for health information: results from the US National Health Interview Survey. J Med Internet Res 17:e106, 2015

3. Asken BM, McCrea MA, Clugston JR, Snyder AR, Houck ZM, Bauer RM: "Playing through it": delayed reporting and removal from athletic activity after concussion predicts prolonged recovery. J Athl Train 51:329-335, 2016

4. Berg GM, Hervey AM, Atterbury D, Cook R, Mosley M, Grundmeyer R, et al: Evaluating the quality of online information about concussions. JAAPA 27:1-8, 2014

5. Broglio SP, Cantu RC, Gioia GA, Guskiewicz KM, Kutcher J, Palm M, et al: National Athletic Trainers' Association position statement: management of sport concussion. J Athl Train 49:245-265, 2014

6. Bryan MA, Rowhani-Rahbar A, Comstock RD, Rivara F: Sports- and recreation-related concussions in US youth. Pediatrics 138:e20154635, 2016

7. Centers for Disease Control and Prevention: Heads up. Managing return to activities. CDC.gov. (https://www.cdc. gov/headsup/providers/return_to_activities.html) [Accessed September 22, 2017]

8. Centers for Disease Control and Prevention: Nonfatal traumatic brain injuries related to sports and recreation activities among persons aged $\leq 19$ years - United States, 2001-2009. MMWR Morb Mortal Wkly Rep 60:1337-1342, 2011

9. Del Vicario M, Bessi A, Zollo F, Petroni F, Scala A, Caldarelli G, et al: The spreading of misinformation online. Proc Natl Acad Sci U S A 113:554-559, 2016

10. Diaz JA, Griffith RA, Ng JJ, Reinert SE, Friedmann PD, Moulton AW: Patients' use of the Internet for medical information. J Gen Intern Med 17:180-185, 2002

11. Elbin RJ, Sufrinko A, Schatz P, French J, Henry L, Burkhart $S$, et al: Removal from play after concussion and recovery time. Pediatrics 138:e20160910, 2016

12. Fendrich H, Pells E: Youth concussion laws pushed by NFL lack bite. Associated Press. January 28, 2015. (http:// www.apnewsarchive.com/2015/AP_Analysis\%3A_Youth_ concussion_laws_pushed_by_NFL_lack_bite/id-87ca43c580 8b4b8dbc8ffb399f6e0970) [Accessed September 22, 2017]

13. Flaherty MR, Raybould T, Jamal-Allial A, Kaafarani HMA, Lee J, Gervasini A, et al: Impact of a state law on physician practice in sports-related concussions. J Pediatr 178:268274, 2016

14. Flores G, Lin H, Walker C, Lee M, Portillo A, Henry M, et al: A cross-sectional study of parental awareness of and reasons for lack of health insurance among minority children, and the impact on health, access to care, and unmet needs. Int J Equity Health 15:44, 2016

15. Fox S: Health Topics. $80 \%$ of Internet Users Look for Health Information Online. Washington, DC: Pew Research Center, 2011 (http://www.pewinternet.org/files/oldmedia/Files/Reports/2011/PIP_Health_Topics.pdf) [Accessed September 22, 2017]

16. Giza CC, Kutcher JS, Ashwal S, Barth J, Getchius TSD, Gioia GA, et al: Summary of evidence-based guideline update: evaluation and management of concussion in sports: report of the Guideline Development Subcommittee of the American Academy of Neurology. Neurology 80:22502257,2013

17. Grool AM, Aglipay M, Momoli F, Meehan WP III, Freedman SB, Yeates KO, et al: Association between early participation in physical activity following acute concussion and 
persistent postconcussive symptoms in children and adolescents. JAMA 316:2504-2514, 2016

18. Harmon KG, Drezner J, Gammons M, Guskiewicz K, Halstead M, Herring S, et al: American Medical Society for Sports Medicine position statement: concussion in sport. Clin J Sport Med 23:1-18, 2013

19. Healthwise: Returning to play after a head injury during a sporting event. WebMD. (http://www.webmd.com/brain/ tc/returning-to-play-after-a-head-injury-during-a-sportingevent-topic\#1) [Accessed September 22, 2017]

20. Kerr ZY, Zuckerman SL, Wasserman EB, Covassin T, Djoko A, Dompier TP: Concussion symptoms and return to play time in youth, high school, and college American football athletes. JAMA Pediatr 170:647-653, 2016

21. Langlois JA, Rutland-Brown W, Wald MM: The epidemiology and impact of traumatic brain injury: a brief overview. J Head Trauma Rehabil 21:375-378, 2006

22. McCrory P, Meeuwisse W, Aubry M, Cantu B, Dvorak J, Echemendia RJ, et al: Consensus statement on concussion in sport-the 4th International Conference on Concussion in Sport held in Zurich, November 2012. Clin J Sport Med 23:89-117, 2013

23. McLendon LA, Kralik SF, Grayson PA, Golomb MR: The controversial second impact syndrome: a review of the literature. Pediatr Neurol 62:9-17, 2016

24. National Federation of State High School Associations: A parent's guide to concussion. NFHS.org. (https://www.nfhs. org/sports-resource-content/a-parents-guide-to-concussion/) [Accessed September 22, 2017]

25. Pfister T, Pfister K, Hagel B, Ghali WA, Ronksley PE: The incidence of concussion in youth sports: a systematic review and meta-analysis. Br J Sports Med 50:292-297, 2016

26. Powell J, Inglis N, Ronnie J, Large S: The characteristics and motivations of online health information seekers: crosssectional survey and qualitative interview study. J Med Internet Res 13:e20, 2011

27. Sports Concussion Institute: Resources. Concussion Treatment.com. (http://concussiontreatment.com/ resources/) [Accessed September 22, 2017]

28. Straus LB, Meehan WP III: Gradual return to play after concussions recommended. MomsTeam.com. (http://www. momsteam.com/health-safety/return-to-play/concussionreturn-to-play-step-by-step-approach-recommended) [Accessed September 22, 2017]

29. Thomas DG, Apps JN, Hoffmann RG, McCrea M, Hammeke T: Benefits of strict rest after acute concussion: a randomized controlled trial. Pediatrics 135:213-223, 2015

30. Thomas DJ, Coxe K, Li H, Pommering TL, Young JA, Smith GA, et al: Length of recovery from sports-related concussions in pediatric patients treated at concussion clinics. Clin J Sport Med [epub ahead of print], 2017

31. West TA, Marion DW: Current recommendations for the diagnosis and treatment of concussion in sport: a comparison of three new guidelines. J Neurotrauma 31:159-168, 2014

32. Zuckerman SL, Zalneraitis BH, Totten DJ, Rubel KE, Kuhn AW, Yengo-Kahn AM, et al: Socioeconomic status and outcomes after sport-related concussion: a preliminary investigation. J Neurosurg Pediatr 19:652-661, 2017

\section{Disclosures}

Dr. Park has served as a consultant for Globus, Medtronic, NuVasive, and Biomet-Zimmer and has received royalties from Globus.

\section{Author Contributions}

Conception and design: Joseph. Acquisition of data: Joseph, Swallow, Willsey. Analysis and interpretation of data: Joseph, Swallow, Willsey. Drafting the article: Joseph, Swallow, Willsey. Critically revising the article: all authors. Reviewed submitted version of manuscript: all authors. Approved the final version of the manuscript on behalf of all authors: Joseph. Statistical analysis: Joseph. Study supervision: Joseph, Park, Szerlip, Broglio.

\section{Correspondence}

Jacob R. Joseph, Department of Neurosurgery, University of Michigan, 1500 East Medical Center Dr., Rm. 3552 TC, Ann Arbor, MI 48109-5338. email: jojacob@med.umich.edu. 